\title{
THE TRANSFER IN HUMANS OF DELAYED SKIN SENSITIVITY TO STREPTOCOCCAL M SUBSTANCE AND TO TUBERCULIN WITH DISRUPTED LEUCOCYTES ${ }^{1,2}$
}

\author{
By H. SHERWOOD LAWRENCE \\ (From the Department of Medicine, New York University College of Medicine, and the Third \\ (NYU) Medical Division, Bellevue Hospital, New York, N. Y.)
}

(Submitted for publication August 2, 1954; accepted October 13, 1954)

It is possible to transfer regularly immunologically specific, generalized skin sensitivity of the delayed type to tuberculin in animals $(1,2)$ and in human subjects $(3,4)$ by means of an injection of leucocytes obtained from sensitive donors.

The cellular transfer system has been applied to the study of the streptococcal hypersensitive state in human subjects $(5,6)$ and the results parallel those obtained with tuberculin as the test material.

In the human subject the induced tuberculin skin sensitivity following leucocytic transfer is, in many respects, similar to that which occurs spontaneously following infection with the tubercle bacillus-namely, it is immunologically specific; it involves the entire skin of the recipient wherever tested; it is frequently as marked in intensity and occasionally maximal skin sensitization of the recipient has been accompanied by a systemic response.

The tuberculin sensitive state following cellular transfer differs from that occurring spontaneously, in that sensitization of some recipients by the former method may be evanescent and disappear entirely in a matter of days or weeks. However, many recipients following cellular transfer may remain sensitized for six to eight months and a few for as long as one year. ${ }^{3}$

A similar parallelism between the spontaneous skin sensitivity to streptococcal materials and that induced following cellular transfer cannot be drawn because there is no clear demonstration in man that the acquisition of positive skin reactions

\footnotetext{
1 Conducted under a contract recommended by the Commission on Streptococcal Diseases, Armed Forces Epidemiological Board, Office of the Surgeon General, U. S. Army.

2 Presented in part at the Forty-Sixth Annual Meeting of the American Society for Clinical Investigation, Atlantic City, New Jersey, May 4, 1954.

8 Observations made on recipients subsequent to publication of References 3 and 5 .
}

to streptococcal materials with age is directly referable to a specific hemolytic streptococcal infection (7).

The known factors which appear to condition the degree and the duration of the sensitive state following transfer, are the degree of sensitivity of the leucocyte donor and the amount of the leucocytes used. Moreover, leucocytes obtained from non-sensitive donors have not induced sensitization of the recipient.

In each of the studies on delayed bacterial sensitivity cited above, the leucocytes used have been intact and viable. In animals treatment of the leucocytes by freezing and thawing has consistently abolished the capacity to transfer delayed sensitivity to tuberculin $(1,2)$ and the treatment of the leucocytes by freezing and thawing $(2,8)$ or distilled water lysis (8) has also abolished the capacity to transfer serum antibody. Recently, successful transfer of contact sensitivity of the delayed type to 2, 4-dinitrochlorobenzene has been reported in animals using leucocytes disrupted by sonic vibration; however, attempts to extend this observation to other delayed sensitivities, including tuberculin sensitivity, were stated to be without success (9).

It is the purpose of this report to present data which indicate in the human, that successful transfer of delayed skin sensitivity to tuberculin and to partially purified preparations of the streptococcal $M$ substance can be achieved regularly with suspensions of leucocyte components liberated, either by repeated freezing and thawing or by distilled water lysis. In addition the treatment of such disrupted leucocyte suspensions with the enzymes desoxyribonuclease (DNase) ${ }^{4}$ or ribonuclease (RNase)," respectively, did not diminish the ca-

4 The abbreviations DNase for desoxyribonuclease, DNA for desoxyribonucleic acid, RNase for ribonuclease, and RNA for ribonucleic acid are used in this report. 
pacity to induce sensitization of the recipient to tuberculin.

\section{MATERIALS AND METHODS}

\section{Isolation of leucocytes}

Detailed descriptions of the technic of leucocyte isolation have been reported $(3,5)$. Briefly, the leucocytes are isolated from the heparinized venous blood of appropriately sensitive donors following the acceleration of the erythrocyte sedimentation rate with bovine fibrinogen, Fraction I (Armour). The supernatant plasma suspension of leucocytes is collected and centrifuged, ${ }^{b}$ the plasma pipetted off and the isolated leucocytes pooled.

Repeated washing of the pooled leucocytes, to elute contaminating erythrocytes and traces of plasma, was not carried out in this study since it was shown in earlier studies that neither erythrocytes, nor plasma, nor serum, but only leucocytes, and only those obtained from markedly sensitive donors, were capable of inducing delayed sensitivity $(3,5)$. Attention is paid to the yield of leucocytes, since it has been observed that if the challenging skin test material is injected at a site remote from the site of leucocyte injection, then at least $0.1 \mathrm{cc}$. of leucocytes are required for the successful transfer of generalized delayed sensitivity $(3,5,6)$. For the experiments to be described 120 to $180 \mathrm{cc}$. of blood is employed with an approximate yield of 0.3 to $0.6 \mathrm{cc}$. leucocytes for each transfer.

\section{Treatment of leucocytes}

The pooled leucocytes are treated by one of the following procedures, and the progress of leucocyte disruption is followed to its completion by parallel giemsa and Feulgen stained (10) smears.

1. Distilled water lysis. For complete lysis of the cell population in a pool of 0.3 to $0.6 \mathrm{cc}$. leucocytes, at least 1 to $2 \mathrm{cc}$. of distilled water for each $0.04 \mathrm{cc}$. leucocytes is necessary. For $0.4 \mathrm{cc}$. leucocytes this would amount to a 10 to $12 \mathrm{cc}$. solution, which is then placed in a $37^{\circ} \mathrm{C}$. water bath for two to four hours with frequent agitation by pipetting to insure adequate contact and to break up the floccules of nucleoprotein.

Microscopic observations made during the course of leucocytolysis indicate that this procedure results initially in the rupture of the cell membrane with leakage of the cytoplasmic components into the supernatant portion of the solution. Subsequently the nuclear membrane ruptures and the nuclear material unravels into skeins of a viscous sediment. The latter takes on a purple color when stained by the Feulgen method and is Feulgenpositive, indicating the presence of highly polymerized Desoxyribonucleic acid (DNA) for which this stain is

The gentle centrifugation, important for the isolation of intact, viable cells, was replaced in this study with centrifugation for 5 minutes at 1,000 RPM and $10 \mathrm{~min}$ utes at 2,600 RPM. specific (11). The cytoplasmic material in the supernatant is colored green and is Feulgen-negative. In the skeins of nucleoprotein sediment there are caught what appear to be Feulgen-negative cytoplasmic ghosts from which the nucleus has been extruded en masse or from which the nuclear material has been leached in the lytic process. There is usually no Feulgen-positive material in the cytoplasmic ghosts; however, in a few, flecks of Feulgen-positive material may remain. The number of what appear to be intact leucocytes or intact round cell nuclei will vary from none, to rare, to one per 50 to 85 high power fields. The nucleoprotein sediment is centrifuged at 1,000 RPM for 15 minutes and resuspended for injection in 2 cc. of the cytoplasmic supernatant. In the experiments detailed in Table $I$, it is this nucleoprotein sediment resuspended in only $2 \mathrm{cc}$. of cytoplasmic solution which is injected into the negative recipient. In the experiments detailed in Table II, it is the remainder of the cytoplasmic supernatant solution which is injected into the negative recipient. It contains occasional fragments of Feulgen-positive nuclear material but not any intact cells or cytoplasmic ghosts.

2. Freezing and thazeing. The pooled leucocytes are resuspended in $3 \mathrm{cc}$. normal saline and frozen in a dry-ice90 per cent alcohol solution and are kept frozen solid for two hours before the first thawing in a $37^{\circ} \mathrm{C}$. water bath. The leucocytes are then alternately rapidly frozen for 10 to 15 minutes and thawed in the $37^{\circ} \mathrm{C}$. water bath for 10 to 15 minutes, for a total of 7 to 10 cycles. The end point of each freezing cycle is taken as the time of appearance of a central nipple in the solid mass rather than any rigid time unit. The total number of freezing and thawing cycles for any particular experiment is governed by the microscopic evidence of the completeness of the disruption of the leucocytes. Microscopic observations made during the course of freezing and thawing are similar in most respects to those detailed under distilled water lysis. There are, however, fewer cytoplasmic ghosts noted following this method of disruption.

3. Freesing and thawing plus ensymes. The leucocytes are frozen and thawed as in Section 2 above and then treated for one hour in a $37^{\circ} \mathrm{C}$. water bath with the enzymes desoxyribonuclease (DNase) or ribonuclease (RNase), respectively. Periodic pipetting of the mixture is employed to insure adequate contact between enzyme and substrate.

a) Crystalline pancreatic desoxyribonuclease (DNase) (Worthington $1 \times$ Crystallized, Lot D-424) (12) is dissolved in $10 \mathrm{cc}$. normal saline containing $0.2 \mathrm{cc}$. normal human serum albumin (Cutter) to a concentration of 1 mgm. per cc. equivalent to 120,000 units by viscosimetric assay. The method of viscosimetric assay used is that of Johnson, Goger, and Tillett (13). The leucocytes to be treated with DNase are frozen and thawed in saline to which has been added $100 \mathrm{mgm}$. MgSO. The $\mathrm{Mg}^{++}$ is added to inhibit the citrate ion (bovine fibrinogen contains approximately 40 to 50 per cent citrate) and to activate the DNase (14). One mgm. of DNase $(120,000$ units) is added to the frozen and thawed leucocytes and 
allowed to act for one hour at $37^{\circ} \mathrm{C}$. water bath before injection of the suspension into the recipient. Paralleling the gross change in viscosity following the addition of DNase, the skeins of DNA fibrillae are no longer discernible microscopically and their Feulgen-positive quality is lost. Structures resembling intact leucocytes or round cell nuclei-and were interpreted as such after lysis or freezing and thawing-disappear as do the cytoplasmic ghosts following dissolution of the protective matrix of DNA fibrillae. This would indicate that such structures were neither intact nor viable leucocytes since DNase does not attack living cells (15). It is rare to find an intact, viable leucocyte in DNase treated suspensions.

b) Crystalline ribonuclease (Worthington, Salt and Protease-free) (Lot R-522-4) $(16,17)$ is dissolved in $0.0005 \mathrm{~N} \mathrm{HCl}$ at a concentration of $2 \mathrm{mgm}$. per cc. at a $\mathrm{pH}$ of 2 and to $0.5 \mathrm{cc}$. of this solution is added $0.5 \mathrm{cc}$. Sorenson's buffer solution. The final concentration of ribonuclease is $1 \mathrm{mgm}$. per cc. at a $\mathrm{pH}$ of 7 .

Citrate $(1 \mathrm{cc}$. of 2.5 per cent solution sodium citrate for each 10 cc. blood) is substituted for heparin as the anticoagulant for leucocyte isolation in the ribonuclease experiments since heparin inhibits ribonuclease activity (18). In addition, since the various lots of RNase were found to have $0.1,0.2$, and 0.03 units of DNase activity per milligram of RNase by viscosimetric assay, the pooled leucocytes are suspended in equal volumes $(1.5 \mathrm{cc}$.) of citrate and normal saline, adequate to inhibit the DNase activity (14) before freezing and thawing.

When RNase is added to frozen and thawed leucocyte suspensions no grossly visible change in viscosity occurs. The extracellular DNA remains highly polymerized and Feulgen-positive and the cytoplasmic elements retain the green color on Feulgen stain.

\section{Test materials}

Streptococcal $M$ substance. ${ }^{8}$ Partially purified preparations of $M$ substance prepared from Type I hemolytic streptococcus are used. The method of preparation and the properties of $M$ substance (Lot A15) have been described by Lancefield and Perlmann (19). Lyophilized $M$ substance is dissolved in saline so that each skin test dose in $0.1 \mathrm{cc}$. solution contains $0.01 \mathrm{mgm}$. (equivalent to $1.4 \gamma$ nitrogen) or $0.002 \mathrm{mgm}$. (equivalent to $0.028 \gamma$ nitrogen). The use of the term "M substance" in this report is descriptive and such usage is not meant to imply chemical or immunological purity.

Old tuberculin (O. T.) (Bureau of Laboratories, Department of Health, N. Y. C.) Second test strength1:1000 dilution equivalent to $0.1 \mathrm{mgm}$. tuberculin for each skin test dose of $0.1 \mathrm{cc}$.

Purified protein derivative (PPD) (Parke, Davis \& Company.) Second test strength- equivalent to 0.005 mgm. PPD for each skin test dose of $0.1 \mathrm{cc}$.

\section{Criteria for reading skin tests}

The criteria for reading delayed cutaneous reactions to old tuberculin, purified protein derivative or strepto-

\footnotetext{
- Kindly supplied by Dr. R. C. Lancefield.
}

coccal $M$ substance are the same as those used in earlier studies $(3,5)$ and reactions are designated from 0 to $4 t$, with each $(+)$ equivalent to approximately $10 \times 10 \mathrm{~mm}$. edema and erythema.

\section{Selection of donors and recipients}

1. Streptococcal $M$ substance as the test material. The group is comprised of $10 \mathrm{M}$-positive leucocyte donors with marked delayed skin sensitivity to streptococcal $M$ substance at 24 hours and 48 hours after skin testing and $14 \mathrm{M}$-negative recipients with no cutaneous reaction to the same concentration of $M$ substance on at least two occasions just prior to transfer. The concentration of $M$ substance used for screening the donors and recipients for nine transfers was $0.01 \mathrm{mgm}$. (1.4 $\gamma$ nitrogen) and for four transfers was $0.002 \mathrm{mgm}$. ( $0.028 \gamma$ nitrogen) per skin test dose, respectively. Recipient RiD (Table II) was tested on only one occasion just before transfer. One additional $M$-negative subject was used as a donor for control studies.

The 10 donors and 14 recipients of the leucocytes were patients hospitalized on the wards of the Third (NYU) Medical Division of Bellevue Hospital and ranged in age from 40 to 60 years with four exceptions. The subjects had no clinical or historical evidence of recent hemolytic streptococcal infection or its delayed complications and were not cachectic nor gravely ill.

2. Tuberculin as the test material. The group is comprised of three tuberculin positive donors of leucocytes with marked delayed skin sensitivity to the tuberculin patch test and 21 tuberculin negative recipients with no skin sensitivity to either second strength old tuberculin $0.1 \mathrm{cc}$. $(0.1 \mathrm{mgm}$.) or second strength PPD $0.1 \mathrm{cc}$. $(0.005$ $\mathrm{mgm}$.) on at least two occasions just prior to transfer. The 16 tuberculin negative recipients making up the test and control groups of the enzyme treated leucocyte suspension studies, were negative to both old tuberculin and PPD on two occasions just prior to transfer.

The donors and recipients of the leucocytes in this group were all healthy male medical students who ranged in age from 22 to 26 years. There was one female student nurse in the group. Each of the medical students had been followed by the author for at least two years and many for three years prior to transfer. In each there had been no clinical or roentgenographic evidence of tuberculosis and each negative recipient had remained tuberculin negative when tested on repeated occasions during this period.

The student nurse (MaS) originally tuberculin negative (PPD $0.005 \mathrm{mgm}$.) in March, 1951, was given BCG in April, 1951 and became positive to tuberculin (+++ PPD $0.005 \mathrm{mgm}$.) in June, 1951. When subsequently retested in May, 1952, June, 1953 and October, 1953, prior to transfer, skin reactions to tuberculin (PPD $0.005 \mathrm{mgm}$. and O. T. $1.0 \mathrm{mgm}$.) were negative on each occasion.

\section{Method of transfer}

When the treatment of the leucocytes has been brought to completion (lysis, freeze-thawing, freeze-thawing plus 
enzyme) it is that respective suspension which is injected intradermally and/or subcutaneously into the skin of the shoulder of the respective negative recipient and such depots are not disturbed thereafter. The following day (18 to 24 hours after injection) the skin of the volar surface of the opposite forearm is challenged with the same concentration of the specific testing material to which the recipient had not been sensitive and the leucocyte donor sensitive. Cutaneous reactions to the test material are recorded at 6 hours, 24 hours and 48 hours. The evolution and subsequent course of the induced sensitivity is followed by repeating the skin test at approximately weekly intervals thereafter, until reversion to the original negative state occurs.

In the three transfers done with the cytoplasmic supernatant solution of distilled water lysed leucocytes, the much larger volume is injected intramuscularly in the deltoid or buttock, and the forearm challenged as above.

\section{RESULTS}

\section{Studies with mechanically disrupted leucocytes}

A. Streptococcal $M$ substance as the test material. Partially purified preparations of Streptococcal $M$ substance (Type I) were chosen as a streptococcal test material because of the close resemblance of the behaviour of $M$ substance in the induced $\mathrm{M}$-sensitive state following cellular transfer in humans to the behaviour of tuberculin in tuberculin sensitivity. In particular, intradermal injection of $M$ substance into reactors with cutaneous hypersensitivity produces predominantly delayed skin reactions of the tuberculin type; moreover, repeated skin testing with $M$ substance does not by itself cause the appearance of delayed reactions of the tuberculin type $(5,6)$.

It is to be emphasized that the preparations of $M$ substance used in these studies are only partially purified, and that delayed skin reactions to such preparations may be due to some contaminating material not yet identified. It is possible that the reactions induced in the leucocyte recipients may also be due to trace amounts of a contaminating material, rather than to the $M$ antigen itself (see ViF, Table III).

There has been no attempt here to define the contribution of contaminating materials in the $M$ substance, or of its type specificity, to the delayed reactions observed. While precise, quantitative immunochemical technics can be utilized in the study of antigens having related skin-sensitizing serum antibody systems (20-25), the cellular transfer system is not susceptible to the adaptation of such technics, at this time, since the substance(s) in the leucocyte responsible for the transfer of delayed sensitivity has yet to be identi fied and quantitated.

Therefore, the delayed skin reaction to partially purified preparations of streptococcal $M$ substance is here used as an indicator, and it is the underlying mechanism of the cellular transfer system which is being evaluated.

The results of the transfer of delayed sensitivity

TABLE I

TRANSFER OF DELAYED M -SENSITIVITY WITH LYSED LEUCOCYTES USING THE NUCLEOPROTEIN SEDIMENT IN CYTOPLASMIC SOLUTION.

\begin{tabular}{|c|c|c|c|c|c|c|c|c|}
\hline \multirow{2}{*}{\multicolumn{2}{|c|}{$\begin{array}{l}\text { M-Negative } \\
\text { Recipient }\end{array}$}} & \multirow[t]{2}{*}{$\begin{array}{l}\text { M-Positive } \\
\text { Donor }\end{array}$} & \multirow[t]{2}{*}{$\begin{array}{l}\text { Donor's } \\
\text { Degree } \\
\text { Sensitivity }\end{array}$} & \multirow{2}{*}{$\begin{array}{l}\text { Approx. } \\
\text { Volume } \\
\text { WBC } \\
\text { For } \\
\text { Lysis } \\
\pm \text { cc. }\end{array}$} & \multicolumn{3}{|c|}{$\begin{array}{c}\text { Maximum Cutaneous } \\
\text { M-Sensitivity } \\
\text { In Recipient }\end{array}$} & \multirow{2}{*}{$\begin{array}{l}\text { Duration of } \\
\text { Sensitized State } \\
\text { in Reciplent } \\
\text { Days }\end{array}$} \\
\hline & & & & & \begin{tabular}{l|} 
Days \\
After \\
Transfer
\end{tabular} & \begin{tabular}{|l|}
24 Hrs. \\
After \\
Skin Test
\end{tabular} & \begin{tabular}{|l|}
48 Hrs. \\
After \\
Skin Test
\end{tabular} & \\
\hline \multirow{3}{*}{ NiR } & Exp. 1 & PeB & $3+$ & 0.5 & - & 0 & 0 & - \\
\hline & Exp. 2 & FiT & $4+$ & 0.3 & 14 & $2+$ & $2+$ & 24 \\
\hline & Exp. 3 & TaS & $4+$ & 0.5 & 1 & $2+$ & $2+$ & 23 \\
\hline \multirow{3}{*}{ HeI } & Exp. 4 & PeB & $3+$ & 0.5 & - & 0 & 0 & - \\
\hline & Exp. 5 & FiT & $4+$ & 0.08 & - & 0 & 0 & - \\
\hline & Exp. 6 & FiT & $4+$ & 0.4 & 9 & $3+$ & $1+$ & $>21$ \\
\hline InG & Exp. 7 & JoC & $4+$ & 0.6 & 2 & $4+$ & $4+$ & $>7$ \\
\hline Anc & Exp. 8 & InG & $4+$ & 0.5 & 2 & $2+$ & $1+$ & 5 \\
\hline JoB & Exp. 9 & WiW & $4+$ & 0.3 & 2 & $3+$ & $1+$ & 17 \\
\hline
\end{tabular}


to streptococcal $M$ substance in negative recipients of the nucleoprotein sediment in a cytoplasmic solution of lysed leucocytes appear in Table I.

In six consecutive instances the $M$-negative recipients of the nucleoprotein and cytoplasmic components of lysed leucocytes obtained from M-positive donors, developed varying degrees of delayed skin sensitivity to $M$ substance.

Negative recipient $\mathrm{NiR}$ failed to develop sensitivity (Exp. 1) following the injection of lysed leucocytes obtained from a moderately sensitive donor $(\mathrm{PeB})$ yet subsequently developed sensitivity (Exp. 2) following the injection of lysed leucocytes, albeit in a lower dosage, obtained from a markedly sensitive donor (FiT). The induced sensitivity in recipient $\mathrm{NiR}$ had disappeared when retested for the third time, 24 days following the successful transfer of Experiment 2. Sensitivity to $M$ substance in recipient $\mathrm{NiR}$ was induced again (Exp. 3) following the injection of lysed leucocytes obtained from another markedly sensitive donor $(\mathrm{TaS})$ and disappeared again on the retest of the 23rd day after transfer.

A similar failure to develop sensitivity was observed in negative recipient HeI (Exp. 4) when lysed leucocytes from the same moderately sensitive donor $(\mathrm{PeB})$ were used. In addition recipient $\mathrm{HeI}$ (Exp. 5) also failed to develop sensitivity when the lysate of an insufficient volume ( 0.08 cc.) of leucocytes obtained from the markedly sensitive donor (FiT, Exp. 2 above) was deliberately chosen to be given. However, this negative re- cipient (HeI) finally developed M-sensitivity (Exp. 6) following the injection of the lysate from an adequate volume of leucocytes obtained from the same markedly sensitive donor (FiT).

These results confirm the equal importance of a minimum dosage of leucocytes (at least $0.1 \mathrm{cc}$. and preferably 0.2 to $0.3 \mathrm{cc}$. using this method) and a markedly sensitive donor in the successful transfer of delayed sensitivity. Similar results have been observed in earlier studies in the human using intact, viable leucocytes $(3,5)$.

Negative recipient InG (Exp. 7) developed marked sensitivity to $M$ substance within two days after the injection of lysed leucocytes obtained from markedly sensitive donor (JoC). At the height of this maximum response, on the third day after transfer, leucocytes were in turn obtained from this formerly negative recipient (InG), lysed and injected into a second negative recipient AnC (Exp. 8). Two days after this serial transfer AnC developed minimal sensitivity $(2+)$ to $M$ substance which disappeared entirely when tested again at the end of five days.

In an effort to exclude the contribution of the few intact leucocytes observed, along with the possibility of other intact leucocytes hidden in the tangled skeins of nucleoprotein sediment, cytoplasmic supernatant solutions of leucocyte lysates were prepared as described and assessed for the capacity to transfer delayed sensitivity to $M$ substance. The results appear in Table II.

In three consecutive instances the $\mathrm{M}$-negative

TABLE II

TRANSFER OF DELAYED M-SENSITIVITY WITH LYSED LEUCOCYTES USING CYTOPLASMIC SUPERNATAN $\Gamma$ SOLUTION.

\begin{tabular}{|c|c|c|c|c|c|c|c|}
\hline \multirow{3}{*}{$\begin{array}{l}\text { M-Negative } \\
\text { Recipient }\end{array}$} & \multirow{3}{*}{$\begin{array}{l}\text { M-Positive } \\
\text { Donor }\end{array}$} & \multicolumn{2}{|c|}{ Volumes } & \multirow{2}{*}{\multicolumn{3}{|c|}{$\begin{array}{c}\text { Maximum Cutaneous } \\
\text { M-Sensitivity in } \\
\text { Recipient }\end{array}$}} & \multirow{3}{*}{$\begin{array}{l}\text { Duration of } \\
\text { Sensitized State } \\
\text { in Recipient } \\
\text { Days }\end{array}$} \\
\hline & & \multirow{2}{*}{$\begin{array}{l}\text { WBC } \\
\text { Lysed } \\
\pm c c .\end{array}$} & \multirow{2}{*}{$\begin{array}{l}\text { Super- } \\
\text { natant } \\
\text { Injected } \\
\text { cc. }\end{array}$} & & & & \\
\hline & & & & \begin{tabular}{|l|} 
Days \\
After \\
Transfer
\end{tabular} & \begin{tabular}{|l|}
24 Hrs. \\
After \\
Skin Test
\end{tabular} & \begin{tabular}{|l|}
48 Hrs. \\
After \\
Skin Test
\end{tabular} & \\
\hline RiD & $\mathrm{FiT}^{*}$ & 0.3 & 10 & 15 & $3+$ & \pm & $>60$ \\
\hline Thw & JoC* & 0.5 & 8 & 1 & $2+$ & $2+$ & 15 \\
\hline Ayc & wiw & 0.6 & 7 & 9 & $3+$ & $1+$ & $>20$ \\
\hline
\end{tabular}

- The cytoplasmic supernatants of leucocytes from donors FiT and JoC were prepared at the same time and from the same leucocytes that were lysed for use in Exp. 2 and 7 of Table $I$. 
TABLE III

TRANSFER OF DELAYED M-SENSITIVITY WITH LEUCOCYTES DISRUPTED BY FREEZING AND THAWING.

\begin{tabular}{|c|c|c|c|c|c|c|}
\hline \multirow[t]{2}{*}{$\begin{array}{l}\text { M-Substance } \\
\text { Status of } \\
\text { Donor }\end{array}$} & \multirow[t]{2}{*}{$\begin{array}{l}\text { M-Negative } \\
\text { Recipient }\end{array}$} & \multirow{2}{*}{$\begin{array}{l}\text { Approx. } \\
\text { Volume } \\
\text { WBC } \\
\pm \text { cc. } \\
\text { Frozen } \\
\text { and } \\
\text { Thawed }\end{array}$} & \multicolumn{3}{|c|}{$\begin{array}{c}\text { Maximum Cutaneous } \\
\text { M-Sensitivity } \\
\text { in Recipient }\end{array}$} & \multirow{2}{*}{$\begin{array}{l}\text { Duration of } \\
\text { Sensitized State } \\
\text { in Recipient } \\
\text { Months }\end{array}$} \\
\hline & & & \begin{tabular}{l|} 
Days \\
After \\
Transfer
\end{tabular} & $\begin{array}{l}24 \text { hrs. } \\
\text { After } \\
\text { Skin Test }\end{array}$ & \begin{tabular}{|l|}
48 hrs. \\
After \\
Skin Test
\end{tabular} & \\
\hline \multirow{4}{*}{$\begin{array}{l}\text { Positive (AdL) } \\
\text { Positive (JoS) } \\
\text { Positive (CaR) } \\
\text { Positive (JoB) }\end{array}$} & WiJ & 0.4 & 6 & $3+$ & $2+$ & $>10$ \\
\hline & WiL & 0.3 & 14 & $3+$ & $2+$ & $>1.5$ \\
\hline & EdM† & 0.3 & 4 & $4+$ & $4+$ & 8 \\
\hline & EdM & 0.6 & 3 & $1+$ & $1+$ & Unknown \\
\hline \multirow{3}{*}{$\begin{array}{cc}\text { Negative } & \text { Exp. 1 } \\
\text { (BeH) } & \text { Exp. 2 } \\
& \text { Exp. 3 }\end{array}$} & $\mathrm{ViF}$ & 0.7 & 26 & $4+$ & $3+$ & $>5$ \\
\hline & FrK & 0.2 & - & 0 & 0 & - \\
\hline & $\mathrm{BeH}$ & 0.2 & - & 0 & 0 & - \\
\hline
\end{tabular}

† Developed effusion ankle joint coincident with maximal skin sensitivity.

recipients of the cytoplasmic supernatant prepared from the lysed leucocytes of M-positive donors, developed delayed skin sensitivity to $M$ substance.

The results of the transfer of delayed sensitivity to streptococcal $M$ substance in negative recipients of frozen and thawed leucocytes appear in Table III.

In four consecutive instances the $\mathrm{M}$-negative recipients of repeatedly frozen and thawed leucocytes obtained from M-positive donors, developed varying degrees of generalized delayed cutaneous sensitivity to $M$ substance.

One negative recipient (EdM) developed a tender, swollen ankle joint coincident with the development of the induced positive skin reaction to $M$ substance in the forearm one day after transfer. The swelling, warmth and tenderness of the joint increased and on the third day after transfer, $10 \mathrm{cc}$. of fluid was aspirated. The fluid was clear, yellowish, and viscous with rare round cells on smear. During this period the patient was afebrile with a normal leucocyte count, an ESR = $7 \mathrm{~mm}$. (Wintrobe), ASL titre $=75$ units per cc., C-reactive protein negative and serial electrocardiograms were normal. The involvement of the joint receded rapidly after aspiration. Whether this event was related to the induced skin sensitivity to $M$ substance or was a coincidence is not known.
In control studies the attempt to transfer M-sensitivity with frozen and thawed leucocytes obtained from an M-negative donor (BeH, Exp. 1) resulted in the development of marked $M$ sensitivity in one negative recipient (ViF). Since previous attempts to transfer delayed sensitivity with intact viable leucocytes obtained from negative donors were unsuccessful $(3,5)$ this experiment was repeated. Leucocytes were obtained from the same negative donor ( $\mathrm{BeH}$, Exp. 2) frozen and thawed and injected into another negative recipient ( $F r K$ ) who did not subsequently develop M-sensitivity. In addition leucocytes were again obtained from the same negative donor (BeH, Exp. 3) frozen and thawed and injected back into herself $(\mathrm{BeH})$ without the subsequent development of M-sensitivity.

The reason for the exceptional response of recipient $\mathrm{ViF}$ is not known. It may possibly be related to the fact that he received an amount of leucocytes $(0.7 \mathrm{cc}$.) three and one-half times greater than recipients FrK $(0.2 \mathrm{cc}$.) and $\mathrm{BeH}(0.2 \mathrm{cc}$.) and the induced sensitivity observed may be in this instance due to trace amounts of contaminating materials rather than to the $M$ antigen itself, as indicated above.

Thus in thirteen consecutive instances the M-negative recipients of components of leucocytes obtained from M-positive donors and dis- 
TABLE IV

TRANSFER OF DELAYED TUBERCULDN SENSITIVITY WITH LEUCOCYTES DISRUPTED BY FREEZING AND THAWING.

\begin{tabular}{|c|c|c|c|c|c|c|}
\hline \multirow{2}{*}{$\begin{array}{l}\text { Tuberculin } \\
\text { Status } \\
\text { of } \\
\text { Donor }\end{array}$} & \multirow[t]{2}{*}{$\begin{array}{l}\text { Tuberculin } \\
\text { Negative } \\
\text { Recipient }\end{array}$} & \multirow{2}{*}{$\begin{array}{l}\text { Approx. } \\
\text { Volume } \\
\text { WBC } \\
\pm \text { cc. } \\
\text { Frozen } \\
\text { and } \\
\text { Thawed }\end{array}$} & \multicolumn{3}{|c|}{$\begin{array}{l}\text { Maximum Cutaneous } \\
\text { Tuberculin Sensitivity } \\
\text { in Recipient }\end{array}$} & \multirow{2}{*}{$\begin{array}{l}\text { Duration of } \\
\text { Sensitized State } \\
\text { in Reciplent } \\
\text { Months }\end{array}$} \\
\hline & & & $\begin{array}{l}\text { Days } \\
\text { After } \\
\text { Transfer }\end{array}$ & $\begin{array}{l}24 \text { Hrs. } \\
\text { After } \\
\text { Skin Test }\end{array}$ & $\begin{array}{l}48 \text { Hrs. } \\
\text { After } \\
\text { Skin Test }\end{array}$ & \\
\hline Positive (HuC) & Mas & 0.25 & 22 & $4+$ & $3+$ & $>9$ \\
\hline Positive (MaS) & ArC & 0.50 & 49 & $2+$ & $1+$ & $>7$ \\
\hline Positive (EdC) & JoS & 0.25 & 20 & $2+$ & $1+$ & 3 \\
\hline Negative (FrG) & GeE & 0.4 & - & 0 & 0 & - \\
\hline Negative (GeE) & FrG & 0.5 & - & 0 & 0 & - \\
\hline
\end{tabular}

rupted by means of two different technics developed generalized delayed cutaneous sensitivity to partially purified preparations of $M$ substance.

In three consecutive attempts to transfer $M$ sensitivity with disrupted leucocytes obtained from the same $M$-negative donor one was successful and two were not successful.

$B$. Tuberculin as the test material. It was felt desirable to substantiate the observations detailed above with the tuberculin system, since it is the prototype of delayed bacterial hypersensitivity and is a useful control in evaluating the results of transfer studies of the streptococcal hypersensitive state. Therefore, the studies just described using partially purified preparations of the streptococcal $M$ substance as the test material were repeated using tuberculin as the test material. The isolated leucocytes were disrupted by repeated freezing and thawing as described above.

The results of the transfer of tuberculin sensitivity with the components of repeatedly frozen and thawed leucocytes appear in Table IV.

In three consecutive instances the tuberculin negative recipients of repeatedly frozen and thawed leucocytes obtained from tuberculin positive donors, developed varying degrees of generalized delayed cutaneous hypersensitivity to tuberculin.

The first tuberculin negative recipient ( $\mathrm{MaS})$ developed moderate $(2+)$ tuberculin sensitivity when tested six days after an injection of frozen and thawed leucocytes obtained from tuberculin positive donor ( $\mathrm{HuC}$ ) which reached its maximum $(4+)$ when tested on the 22nd day after transfer. At the height of this maximum response on the 22nd day after transfer, leucocytes were in turn obtained from this formerly negative recipient ( $\mathrm{MaS})$, frozen and thawed and injected into a second negative recipient (ArC). Thirty days after this serial transfer ArC developed minimum sensitivity to tuberculin $(t)$ which reached its maximum $(++)$ at the end of 49 days. At present, eight months after transfer ArC's sensitivity is minimal and has just about disappeared.

In two consecutive instances attempts to transfer tuberculin sensitivity to negative recipients (GeE and FrG, Table IV) with frozen and thawed leucocytes obtained from negative donors were unsuccessful.

Thus the observation that components of disrupted leucocytes are capable of transferring delayed cutaneous sensitivity to streptococcal $M$ substance is substantiated when tuberculin is used as the test material.

\section{Enzyme treatment of frozen and thawed leucocytes}

The reproducible nature of the results just described presented the opportunity to introduce additional variables into the donor system with the reassurance afforded by a relatively stable test system.

For this purpose the isolated leucocytes are frozen and thawed in the manner described and to the suspension of leucocyte components a specific enzyme, desoxyribonuclease (DNase) or ribonuclease ( $\mathrm{RNase}$ ), is added. 
TABLE $\mathbf{V}$

TRANSFER OF DELAYED TUBERCULIN SENSTTIVITY WTTH

FROZEN AND THAWED AND ENZYME TREATED LEUCOCYTES.

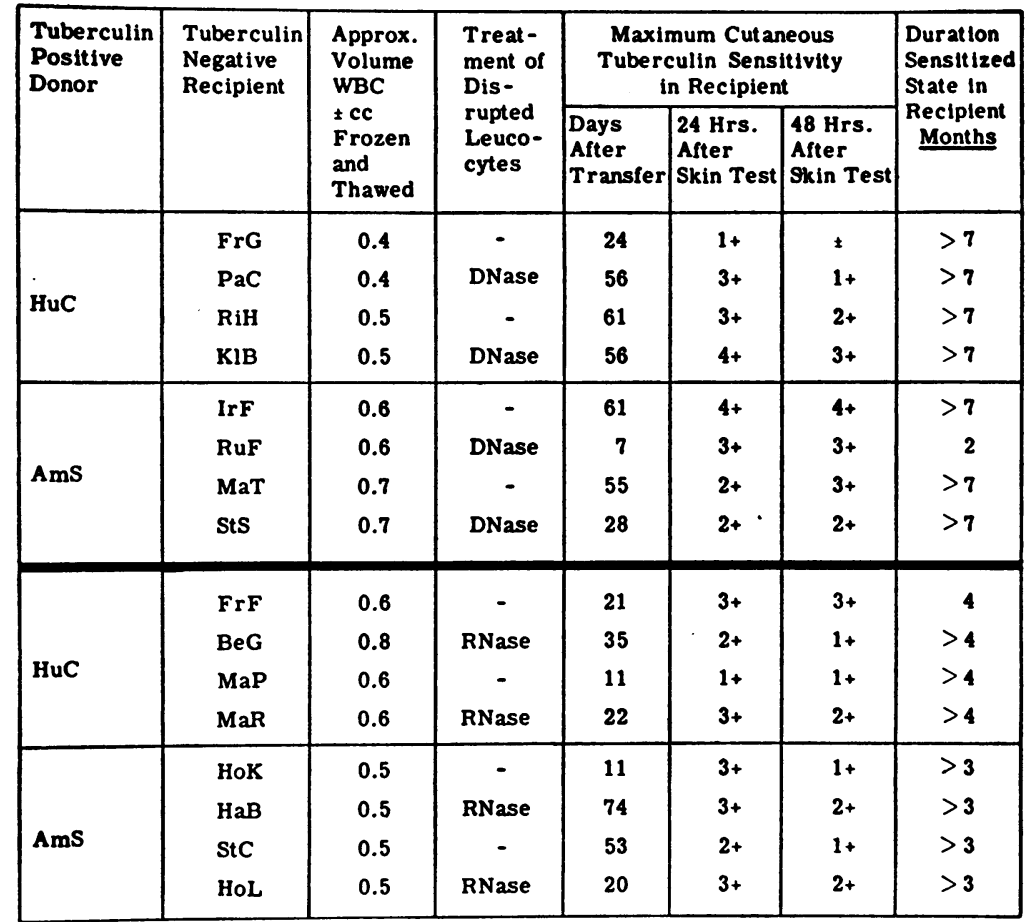

The results of the action of DNase upon the extracellular DNA can be interpreted with some confidence, since the Feulgen stain is highly specific and permits concrete visual evidence of depolymerization of all available DNA (11). There is not such a reliable histochemical technique for RNA and its depolymerization, hence there is some uncertainty about the extent and degree of depolymerization of the RNA in these experiments. This reservation exists despite the advantage taken of all precautions known to be propitious for the action of $\operatorname{RNase}(18,26)$. In addition, a residue of RNA is known to remain undigested even when a large excess of RNase is employed (26).

For this group of experiments sixteen additional tuberculin negative recipients were selected and had prepared for them on different occasions, suspensions of the constituents of repeatedly frozen and thawed leucocytes obtained from one of two tuberculin positive donors. Each of eight alternate recipients received an injection of such untreated suspensions; of the remainder, four recipients received DNase treated suspensions and four recipients received RNase treated suspensions. The results are summarized in Table $\mathrm{V}$.

In sixteen consecutive instances each negative recipient developed generalized delayed cutaneous tuberculin hypersensitivity and the pattern of response appeared similar in the untreated and enzyme treated groups. Of the control group of eight recipients, three became moderately and five markedly sensitive to tuberculin. Of the DNase treated group of four, one became moderately and three markedly sensitive to tuberculin, and of the RNase treated group of four, one became moderately and three markedly sensitive to tuberculin.

\section{Comparison of sensitivity induced with disrupted leucocytes to that induced with intact leucocytes}

In each instance described above the induced delayed sensitivity to $M$ substance or to tuberculin in negative recipients following the injection of disrupted leucocytes is similar in most respects to that observed in human subjects following the injection of intact, viable leucocytes $(3,5)$. and to that seen in the spontaneously sensitive individual (6). 
The Distribution of Transferred

Sensitivity to Tuberculin and

Streptococcal Materials

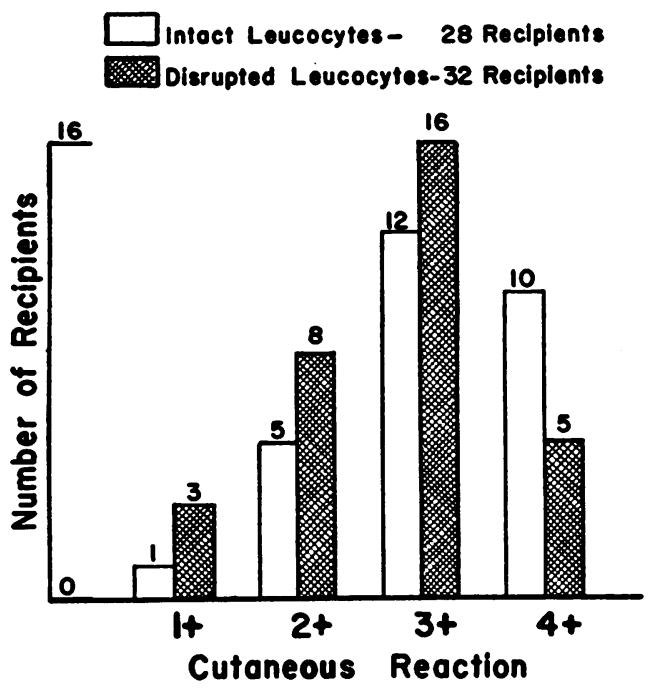

FIGURE 1.

One aspect of this similarity is graphically represented in Figure 1 which plots the distribution in terms of degree of sensitivity achieved in 60 consecutive recipients of the transfer of delayed sensitivity in humans, where the results in 28 recipients ${ }^{7}$ using intact, viable leucocytes $(3,5)$ are compared with the results in the 32 recipients of this report using disrupted cells.

\section{DISCUSSION}

With the finding that it is regularly possible in the human to transfer bacterial sensitivity of the delayed tuberculin type by means of the components of disrupted leucocytes, it became feasible to attempt identification of the particular leucocyte component(s) concerned in the tissue sensitization process and gain some clue to the mechanism whereby sensitization is achieved. A step in this direction is the application of enzymatic techniques to selectively eliminate one cellular component from the whole at a time.

The sequence of events following cellular transfer with intact or disrupted leucocytes, suggests

7 Unpublished results in two recipients of the transfer of M-sensitivity (each graded $4+$ ) using lysed leucocytes which were incompletely disrupted are included among the "intact" leucocyte group. that this system may have analogies to other biological systems where self-replication of a foreign material is fostered by the cells of the host. Examples of the latter are to be found in the behaviour of the DNA of the pneumococcal transforming principle $(27,28)$ and in the intracellular replication of viral particles in microbial (29), plant (30) and animal cells (31-34). This similarity is suggested by the observation that relative to the considerable cell mass of the adult human recipient, an infinitesimal amount of donor cell material is capable of altering the reaction capacity of the entire skin surface of the recipient for periods in many instances as long as six to eight months and in a few as long as one year. This response has heretofore been achieved only following prolonged, intimate contact with considerable numbers of the specific bacterium (tubercle bacillus, streptococcus) and the tissues of the host (6).

Moreover, unlike the passive transfer of preformed serum antibody the transfer of leucocytes, intact or disrupted, appears to require the active participation of the recipient before maximum and sustained effects may be achieved. Although the response is variable for each recipient, a review of the data collected in this and earlier studies $(3,5)$, comprising a total of 60 successful individual transfers, indicates that two broad, but distinct, temporal patterns of recipient response exist following the transfer of delayed sensitivity. The patterns are schematically represented in Figure 2.

It will be recalled that all recipients are skintested within a day after cellular transfer and thereafter at approximately weekly intervals. In this diagram the magnitude $(0$ to $4+)$ of the reaction to each individual skin test is plotted

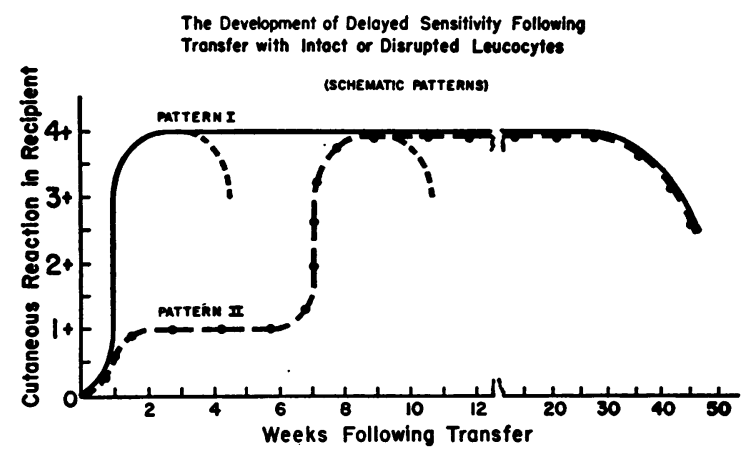

Figure 2. 
against the time of application of that skin test following cellular transfer.

The recipients with a response which approximates that shown in Pattern I exhibit maximum sensitivity $(4+)$ to the first or second skin test, applied within a day or a week following transfer, The recipients with a response which approximates that shown in Pattern II exhibit sensitivity early, as in Pattern I, but the reactions to each of the skin tests are minimal $(1+)$, for intervals which vary from two to six to eight weeks following transfer. At the end of this variable lag period there is an abrupt appearance of maximum sensitivity $(4+)$ in response to the respective weekly skin test. In both patterns, maximum sensitivity may be evanescent and disappear entirely, or it may gradually wane in intensity when the recipient is subsequently retested, or it may persist for prolonged periods on subsequent retesting, as indicated in Figure 2. Since this sequence of events does not occur in relation to the application of one skin test, but represents a progressive change in the magnitude of the response of the recipient to successive skin tests, it differs from the phenomenon described by Andrewes, Derick, and Swift (35), where a delayed flare-up of an intradermal reaction occurs in the same skin test site with the passage of time.

The observation of two distinct temporal patterns following the transfer of an immunological response suggests that two different substances or different phases of the same substance, each related to a separate pattern of response, may be present in the leucocytes of the donor.

The substance which contributes to the development of Pattern I would appear to be more completely preformed and readily available in amounts sufficient to cause the negative recipient to respond rapidly in this early phase (one day to one week), in what appears to be a "passive" manner, to the test material. Whereas the substance which contributes to the development of Pattern II would appear to be less completely preformed and less readily available since it requires longer residence (two to six to eight weeks), presumably intracellularly, in the recipient before it becomes capable of causing the appearance of the sudden burst of maximal sensitivity. Whether the substance postulated for Pattern I is a more or less completely preformed antibody or an antibody precursor, and that postulated for Pattern II an antigen, in the sense that it has resulted from the interaction between the antigens of the intact bacterial cell and the tissues of the donor, is not known.

An alternative possibility is that there may be only one substance which is handled by different mechanisms in the recipient.

In both patterns of response the prolonged duration of sensitization observed in many recipients would suggest a mechanism quite different from that which is involved in the transient effects and early catabolism of preformed serum antibody following passive transfer, presumably incident to its extracellular residence in the recipient.

Because of the difference in behaviour from preformed serum antibody and from evidence, as yet only suggestive, afforded by two attempts at serial transfer, it is reasonable to suppose that this substance(s) may be taken up by the cells of the recipient which are concerned with the production of delayed sensitivity and in turn alter their metabolism for variable periods, perhaps in a manner similar to that by which the specific bacterium, some time in the past, altered the metabolism of the cells of the donor in this regard.

Hence, our attempts to identify the components of the leucocyte responsible for this effect have focussed on the cell components of the donor with self-replicating potentialities. Assuming the substance responsible for the transfer of delayed sensitivity was the DNA of the nucleus, then treatment with the enzyme DNase might be expected to abolish this capacity and similarly if assumed to be the RNA, then treatment with RNase might be expected to abolish it. These assumptions were tested as reported and the results were negative.

From such negative results, conclusions are limited and tentative; however, it would appear that the mechanism responsible for the transfer of delayed sensitivity does not depend upon the highly polymerized DNA or highly polymerized RNA components of the donor cell. The working hypothesis detailed above, however, retains its usefulness in aiding the design of further experimental tests of its validity. 


\section{SUMMARY AND CONCLUSIONS}

1. In 32 consecutive instances it has been possible in human subjects to transfer skin sensitivity of the delayed bacterial type to tuberculin and to partially purified preparations of the streptococcal $M$ substance, with components of disrupted leucocytes obtained from sensitive donors and prepared by two different techniques of disruption.

This was accomplished with streptococcal $M$ substance as the test material using leucocytes prepared by : a) Distilled water lysis, and b) freezing and thawing; and accomplished with tuberculin as the test material using leucocytes prepared by : a) Freezing and thawing, b) freezing and thawing plus DNase, and c) freezing and thawing plus RNase.

2. With one exception, delayed sensitivity did not develop in five negative recipients when the disrupted leucocytes were obtained from donors without sensitivity to the test material.

3. The induced delayed sensitivity to tuberculin and to streptococcal $M$ substance in negative recipients following the injection of components of disrupted leucocytes is similar, in most respects, to that which has been observed in humans following the injection of intact viable leucocytes.

4. Treatment of disrupted leucocyte suspensions with the enzymes DNase or RNase did not diminish the capacity to transfer delayed sensitivity.

5. A working hypothesis concerning the mechanism whereby the transfer of delayed sensitivity may be mediated, is discussed.

\section{ACKNOWLEDGMENT}

The author wishes to acknowledge the excellence of the technical assistance which he has received from $\mathrm{Mr}$. Louis Mazzola.

\section{REFERENCES}

1. Chase, M. W., The cellular transfer of cutaneous hypersensitivity to tuberculin. Proc. Soc. Exper. Biol. \& Med., 1945, 59, 134.

2. Chase, M. W., Immunological reactions mediated through cells in The Nature and Significance of the Antibody Response. A. M. Pappenheimer, Jr., Ed., (New York Academy of Medicine. Section on microbiology. Symposium No. 5), New York, Columbia University Press, 1953, p. 156.

3. Lawrence, H. S., The cellular transfer of cutaneous hypersensitivity to tuberculin in man. Proc. Soc. Exper. Biol. \& Med., 1949, 71, 516.
4. Urbach, F., Sones, M., and Israel, H. L., Passive transfer of tuberculin sensitivity to patients with sarcoidosis. New England J. Med., 1952, 247, 794.

5. Lawrence, H. S., The cellular transfer in humans of delayed cutaneous reactivity to hemolytic streptococci. J. Immunol., 1952, 68, 159.

6. Lawrence, H. S., Transfer of skin reactivity to streptococcal products in Streptococcal Infections. M. McCarty, Ed., (New York Academy of Medicine. Section on microbiology. Symposium, No. 7), New York, Columbia University Press, 1954, p. 143.

7. Mackenzie, G. M., and Hangar, F. M., Jr., Allergic reactions to streptococcus antigens. J. Immunol., 1927, 13, 41.

8. Harris, S., Harris, T. N., and Farber, M. B., Studies on the transfer of lymph node cells. I. Appearance of antibody in recipients of cells from donor rabbits injected with antigen. J. Immunol., 1954, 72, 148.

9. Jeter, W. S., Tremaine, M. M., and Seebohm, P. M., Passive transfer of delayed hypersensitivity to 2, 4-Dinitrochlorobenzene in guinea pigs with leucocytic extracts. Proc. Soc. Exper. Biol. \& Med., 1954, 86, 251.

10. Sherry, S., Johnson, A., and Tillett, W. S., The action of streptococcal desoxyribose nuclease (streptodornase), in vitro and on purulent pleural exudations of patients. J. Clin. Invest., 1949, 28, 1094.

11. Ris, H., and Mirsky, A. E., Quantitative cytochemical determination of desoxyribonucleic acid with the Feulgen nucleal reaction. J. Gen. Physiol., 1949, 33, 125.

12. Kunitz, M., Crystalline desoxyribonuclease. I. Isolation and general properties, spectrophotometric method for the measurement of desoxyribonuclease activity. J. Gen. Physiol., 1949, 33, 349.

13. Johnson, A. J., Goger, P. R., and Tillett, W. S., The intravenous injection of bovine crystalline pancreatic desoxyribonuclease into patients. J. Clin. Invest., 1954, 33, 1670.

14. McCarty, M., Purification and properties of desoxyribonuclease isolated from beef pancreas. J. Gen. Physiol., 1945, 29, 123.

15. Johnson, A. J., Cytological studies in association with local injections of streptokinase-streptodornase into patients. J. Clin. Invest., 1950, 29, 1376.

16. Kunitz, M., Crystalline ribonuclease. J. Gen. Physiol., 1941, 24, 15.

17. McDonald, M. R., A method for the preparation of "protease-free" crystalline ribonuclease. J. Gen. Physiol., 1948, 32, 39.

18. Roth, J. S., Ribonuclease. II. Activators and inhibitors for ribonuclease. Arch. Biochem. \& Biophysics, 1953, 44, 265.

19. Lancefield, R. C., and Perlmann, G. E., Preparation and properties of type-specific $M$ antigen isolated from a group A, type 1 hemolytic streptococcus. J. Exper. Med., 1952, 96, 71. 
20. Vaughan, J. H., and Kabat, E. A., Studies on the antibodies in rabbit antisera responsible for sensitization of human skin. I. The role of impurities in crystalline egg albumin in stimulating the production of skin-sensitizing antibody. J. Exper. Med., 1953, 97, 821.

21. Vaughan, J. H., and Kabat, E. A., Studies on the antibodies in rabbit antisera responsible for sensitization of human skin. II. Characteristics of antisera to egg white and to conalbumin. J. Allergy, 1954, 25, 387.

22. Kuhns, W. J., and Pappenheimer, A. M., Jr., Immunochemical studies of antitoxin produced in normal and allergic individuals hyperimmunized with diphtheria toxoid. I. Relationship of skin sensitivity to purified diphtheria toxoid to the presence of circulating, non-precipitating antitoxin. J. Exper. Med., 1952, 95, 363.

23. Kuhns, W. J., and Pappenheimer, A. M., Jr., Immunochemical studies of antitoxin produced in normal and allergic individuals hyperimmunized with diphtheria toxoid. II. A comparison between the immunological properties of precipitating and non-precipitating (skin-sensitizing) antitoxins. J. Exper. Med., 1952, 95, 375.

24. Lawrence, H. S., and Pappenheimer, A. M., Jr., Immunization of adults with diphtheria toxoid. I. Immunological properties of formalinized diphtherial protein fractions from culture filtrates. Am. J. Hyg., 1948, 47, 226.

25. Pappenheimer, A. M., Jr., and Lawrence, H. S., Immunization of adults with diphtheria toxoid. II. An analysis of the pseudoreactions to the Schick Test. Am. J. Hyg., 1948, 47, 233.

26. Laskowski, M., Nucleolytic enzymes in The Enzymes. J. B. Sumner and K. Myrbäck, Eds., New York, Academic Press, 1951, vol. 1, p. 956.

27. Avery, O. T., MacLeod, C. M., and McCarty, M., Studies on the chemical nature of the substance inducing transformation of pneumococcal types.
Induction of transformation by a desoxyribonucleic acid fraction isolated from pneumococcus type III. J. Exper. Med., 1944, 79, 137.

28. Hotchkiss, R. D., Transfer of penicillin resistance in pneumococci by the desoxyribonucleate derived from resistant cultures. Cold Spring Harbor Symposia on Quantitative Biology, 1951, vol. 16, p. 457.

29. Kozloff, L. M., Origin and fate of bacteriophage material. Cold Spring Harbor Symposia on Quantitative Biology, 1953, vol. 18, p. 209.

30. Bawden, F. L., and Pirie, N. W., Virus multiplication considered as a form of protein synthesis in The Nature of Virus Multiplication. (Society for general microbiology. Symposium No. 2.) England, Cambridge University Press, 1953, p. 20.

31. Hoyle, L., The multiplication of the influenza virus in relation to the general problem of biological multiplication in The Nature of Virus Multiplication. (Society for general microbiology. Symposium No. 2.) England, Cambridge University Press, 1953, p. 225.

32. Brachet, J., The role of the nucleus and the cytoplasm in synthesis and morphogenesis. Structural aspect of cell physiology. Symposia of the Society for Experimental Biology. New York, Academic Press, 1952, vol. 6, p. 173.

33. Chantrenne, H., Problems of protein synthesis in The Nature of Virus Multiplication. (Society for general microbiology. Symposium No. 2.) England, Cambridge University Press, 1953, p. 1.

34. Haurowitz, F., and Crampton, C. F., The role of the nucleus in protein synthesis. Experimental Cell Research, Supplement 2, 1952, p. 45.

35. Andrewes, C. H., Derick, C. L., and Swift, H. F., The skin response of rabbits to non-hemolytic streptococci. I. Description of a secondary reaction occurring locally after intradermal inoculation. J. Exper. Med., 1926, 44, 35. 